
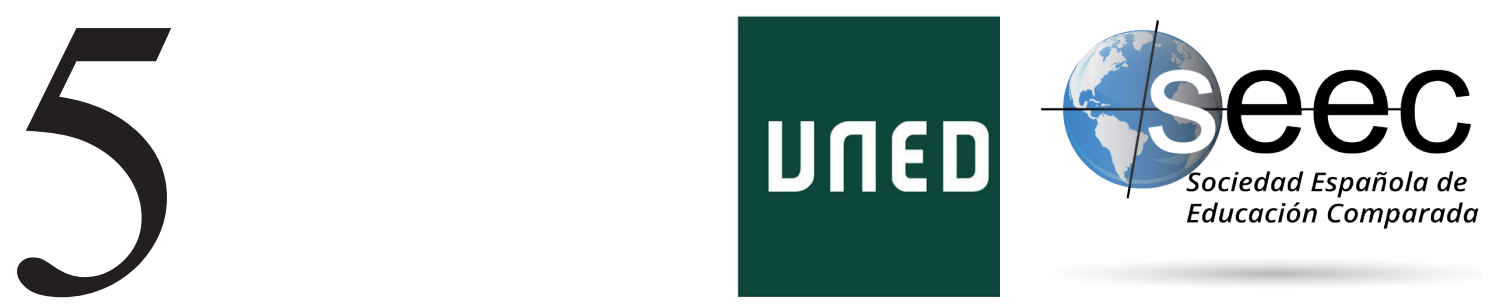

\title{
Universidad Iberoamérica 2030:la propuesta de la OEI para la educación superior en Iberoamérica
}

University Iberoamerica 2030: a proposal by the OEI for the Iberoamerican higher education

\section{Ana Capilla Casco*}

DOI: $10.5944 /$ reec.37.2021.27715

Recibido: 15 de junio de 2020 Aceptado: 29 de julio de 2020

\footnotetext{
*Ana Capilla Casco: es coordinadora de Educación Superior, ETP y Ciencia de la Organización de Estados Iberoamericanos para la Educación, la Ciencia y la Cultura (OEI), y profesora de Relaciones Internacionales en la Universidad Francisco de Vitoria. Datos de contacto: e-mail: educacion_superior@oei.es
} 


\title{
Resumen
}

La educación superior es una de las áreas prioritarias de acción de la OEI actualmente, de acuerdo al mandato recibido por los ministros de educación iberoamericanos. El crecimiento de la matrícula universitaria y la importancia de la educación superior para superar la denominada «trampa de la productividad» que sufre especialmente América Latina, explican este interés. Así como la necesidad cada vez más acuciante de hacer realidad un objetivo largamente ansiado en la región: la construcción de un espacio iberoamericano de educación superior e investigación. Todos estos elementos forman parte de la estrategia Universidad Iberoamérica 2030 que la OEI ha proyectado para la próxima década, y que marcan un camino claro para las instituciones de educación superior.

Palabras clave: universidad; investigación; Agenda 2030; armonización.

\begin{abstract}
Higher education is one of the top priority OEI's lines of action, according to the mandate granted by the Iberoamerican Education ministers to the organization. This is explained by the increasing number of university students and the fact higher education is key to overcome the "productivity trap" the Latin American countries suffered specially. And the every time more pressing need of achieving a long time desired aim: to build an Iberoamerican area of higher education and research. All these elements are integrated in the strategy University Iberoamerica 2030 planned by the OEI for the next decade, and which mark a clear path for the higher education institututions.
\end{abstract}

Keywords: university; research; Agenda 2030; harmonization. 


\section{La cooperación en Iberoamérica: el papel de la educación superior}

En 2019 la Organización de Estados Iberoamericanos para la Educación, la Ciencia y la Cultura (OEI) celebró su 70 aniversario. Esta efeméride ha coincidido con la llegada de un nuevo secretario general a la Organización y, en consecuencia, con el inicio de una nueva etapa que aspira a la máxima lampedusiana de que todo cambie para que todo siga igual.

Todo tiene que cambiar porque Iberoamérica ha experimentado estas pasadas décadas profundas transformaciones en todos los órdenes, también, y de manera muy especial, en la educación superior.

Según el Observatorio de la Ciencia, la Tecnología y la Sociedad de la OEI (OCTSOEI) ${ }^{1}$, en 2017 la matrícula de educación superior en Iberoamérica superó la barrera de los 30 millones de estudiantes. Una cifra que se ha alcanzado gracias a un crecimiento anual sostenido desde 2010 del $3.7 \%$. Aunque los datos concretos de cada país difieren bastante entre sí, según la incidencia en cada uno de ellos por tres factores: las distintas etapas que cada uno atraviesa en su transición demográfica; las tasas de graduación en el nivel medio; y, las políticas de acceso y diferenciación institucional. Así, la matrícula se ha expandido a un ritmo notable en Perú, México, Uruguay y Bolivia, pero ha decrecido en Cuba y Portugal. De manera que, según la clasificación de Martin Trow, Argentina, Chile, España y Uruguay ya se ubican en la etapa de universalización, con tasas brutas de educación superior (TBEs) por encima del $50 \%$. El resto de los países de Iberoamérica está atravesando la etapa de masificación, la cual tiene lugar cuando la TBEs es mayor del $15 \%$ pero menor del $50 \%$. Honduras fue el país iberoamericano con una menor cobertura: 16,2 \% en el año 2017 (OCTS-OEI, 2019a).

En líneas generales, se aprecia que las mayores TBEs se corresponden con los países de la región con más crecimiento económico, medido el mismo en Producto Interior Bruto (PIB), con excepciones como la de Argentina (OCTS-OEI, 2019a), país en el que la gratuidad de la educación superior explica su elevada matrícula. Sin embargo, como viene advirtiendo la Organización para la Cooperación y el Desarrollo Económico (OCDE) y la Comisión Económica para América Latina y el Caribe (CEPAL), en el informe anual que publican sobre las perspectivas económicas de América Latina², el desarrollo de un país no se puede medir solo en datos macroeconómicos. A pesar de que los países de la región han experimentado un considerable progreso social y económico desde el año 2000, se enfrentan a serios obstáculos que limitan su capacidad de seguir creciendo y generando prosperidad.

A efectos de este artículo, de las cuatro trampas estructurales a las que se enfrentan los países de renta media de América Latina, destaca especialmente la trampa de la productividad, muy directamente relacionada con la educación superior, tanto la universitaria como la técnica. Sirva como ejemplo el hecho de que la productividad laboral

1 El Observatorio de la Ciencia, la Tecnología y la Sociedad de la OEI fue creado en 2008 con la misión de desarrollar un programa de estudios estratégicos en Ciencia, Tecnología y Sociedad que indague en las fronteras de la ciencia y de las demandas sociales de los pueblos de Iberoamérica. Se encarga de coordinar la Red de Indicadores de Ciencia y Tecnología (RICYT) y la Red IndicES de Educación Superior, integradas por las Oficinas de Estadística de los Ministerios de Educación y Ciencia de los países iberoamericanos. Ver https://observatoriocts.oei.org.ar/

2 Más conocido por sus siglas en inglés, LEO (Latin America Economic Outlook). 
en la región equivale al 40 \% de la de la Unión Europea. Pero en estos momentos en que las medidas sanitarias adoptadas para combatir el covid-19, especialmente el confinamiento, dibujan un escenario económico tan complicado en la región, resulta inevitable mencionar también la trampa de la vulnerabilidad social (OCDE et al., 2019). Esta afecta a todos aquellos, que durante el periodo de crecimiento económico han tenido acceso a empleos de mala calidad o informales y que, por tanto, carecen de protección laboral, de salud o de pensiones. Según datos de la CEPAL, más de la mitad de los trabajadores de la región, el $53 \%$, se encuentran en esta situación, por lo que calculan que 28 millones de personas pueden caer en la pobreza solo en el presente año, con lo que la pobreza en la región alcanzaría el 34.7\% (CEPAL, 2020).

Todo ello viene a confirmar que las cifras de crecimiento económico de América Latina escondían una realidad frágil, que el COVID-19 está exponiendo en toda su crudeza. La OEI, como organismo decano de la cooperación iberoamericana, ha sido testigo directo de los efectos que ha tenido en la región el hecho de que la mayoría de los países se «graduaran» y dejaran de ser elegibles para la Ayuda Oficial al Desarrollo (AOD). América Latina ha sido en gran medida el laboratorio de una nueva política de cooperación, la cooperación con los países de renta media, en la cual los proyectos basados en los abundantes recursos económicos de tiempos pasados han sido sustituidos por otros altamente especializados en áreas estratégicas para el desarrollo de estos países. Un desarrollo que, en plena $4^{\mathrm{a}}$ revolución industrial y economía del conocimiento, es altamente dependiente de la educación; en especial, de la educación superior y la ciencia. El protagonismo que esta última está adquiriendo en estos momentos, en que nos enfrentamos a una pandemia global, viene a confirmar que este es el camino a seguir. Que el futuro de Iberoamérica depende de su capacidad de generar y compartir conocimiento. Es así como se alcanzará el objetivo de desarrollo sostenible que inspira la Agenda 2030 de Naciones Unidas, referencia compartida por todos los que estamos apelados a la consecución de los Objetivos de Desarrollo Sostenible que contempla.

De manera que, como se señalaba al comienzo de esta introducción, son muchos los cambios que una organización septuagenaria como la OEI ha tenido que afrontar en los últimos meses, como garantía de que todo sigue igual, esto es, que la OEI continúa siendo relevante y útil a los países iberoamericanos. Porque en un escenario político, económico y social tan complicado como el que se ha esbozado, solo los organismos capaces de aportar y contribuir a paliar los efectos secundarios tan perniciosos que nos va a dejar la pandemia, podrán seguir cumpliendo años.

\section{2. ¿Qué es y para qué sirve un espacio compartido de educación superior y ciencia?}

En septiembre de 2018 los ministros de Educación iberoamericanos, reunidos en el $77^{\circ}$ Consejo Directivo de la OEI, aprobaron el programa presupuesto de esta Organización para el bienio 2019-2020. Este incluye por primera vez un capítulo dedicado a Educación Superior, que gira en torno a un objetivo largamente deseado en la región: la creación de un espacio compartido de educación superior e investigación científica.

En un contexto como el que se ha explicado en el apartado anterior, en el que resulta imprescindible consolidar patrones de crecimiento y desarrollo intensivos en conocimiento, tecnologías e innovación, la construcción de un espacio de tales características 
se revela como una herramienta estratégica de cooperación. La pandemia que estamos sufriendo demuestra hasta qué punto nuestras sociedades necesitan investigación avanzada y profesionales cualificados, desde sanitarios que atiendan a los contagiados, hasta ingenieros que pongan en marcha en cuestión de días infraestructuras hospitalarias en las que atenderlos o fabriquen respiradores para los enfermos de cuidados intensivos. En los próximos años es ilusorio pensar que los países iberoamericanos, cuyas cuentas públicas van a atravesar serias dificultades, van a poder afrontar de manera aislada las demandas de sus sistemas de educación superior y científico. Por lo tanto, avanzar hacia su armonización a nivel regional es un objetivo más necesario ahora que nunca.

El Espacio Europeo de Educación Superior (EEES), que arrancó en 1998 con la declaración de La Sorbona, es la referencia por excelencia a estos efectos. Si bien, es importante también recordar, que el EEES forma parte de un ambicioso proyecto de integración regional supranacional que nació en Europa en los años 50, en las postrimerías de la Segunda Guerra Mundial. De hecho, el EEES propicia una armonización muy profunda de los sistemas universitarios europeos con el propósito de favorecer la movilidad de estudiantes y, de modo muy especial, de egresados universitarios. Porque resultaba indispensable el reconocimiento de títulos y cualificaciones por parte del resto de países europeos para garantizar la libertad de establecimiento, sin la cual no podía haber un auténtico Mercado Único

Los beneficios del conocido como proceso de Bolonia en estas dos décadas van mucho más allá de su contribución al mercado interior. Ha creado las condiciones idóneas, gracias al reconocimiento de estudios y títulos, haciendo posible la movilidad de más de cinco millones de estudiantes europeos en el marco del programa Erasmus. Son muchas las ventajas que se derivan de estos intercambios a nivel académico, también en lo que se refiere al futuro laboral de los participantes: dos de cada tres estudiantes descubren nuevas metodologías de estudio y trabajo durante su estancia en su universidad de destino; y hasta uno de cada cuatro reorienta su formación para estudiar algo que le resulta más interesante. El 8o \% de los Erasmus de primer ciclo deciden continuar con una formación; y, el 8o \% de los que deciden ir al mercado laboral encuentran trabajo en los tres primeros meses después de su graduación. Estos efectos positivos son aún mayores en los jóvenes procedentes de los Estados miembros con menor PIB, o de países asociados al programa (Comisión Europea, 2019).

Por si esto no fuera suficiente, el programa Erasmus está considerado como el gran promotor del sentimiento europeo. Después de su movilidad, el $90 \%$ de los estudiantes reconocen que la Unión Europea es necesaria y que ser europeo es un valor añadido. La experiencia de estudiar en otro país de la UE propicia un sentimiento de afección a Europa entre los Erasmus, ya que no solo se sienten parte de la familia europea, sino que muchos incluso han fundado su propia familia europea ${ }^{3}$.

Los beneficios de la movilidad académica son evidentes y no solo para los europeos. Por ese motivo el número de estudiantes de educación superior que emprendieron estudios en otro país entre 2012 y 2017 ha crecido espectacularmente a nivel global: del 2,05 \% del total de los estudiantes de educación superior desplazados internacionalmente a un 2,30 \% (en cifras: de 4 millones a 5 en solo cinco años). En América Latina y Caribe, sin embargo, las cifras son mucho más modestas: se ha pasado del 1,09 \% al $1,14 \%$ (en cifras, de 258 mil estudiantes a 312 mil). Un incremento tan pequeño que hace que sea la segunda región del mundo en el que los estudiantes disfruten de menos

3 En 2014 la Comisión calculó que habían nacido un millón de bebés europeos de parejas Erasmus. 
intercambios académicos. Y más de la mitad, el $54 \%$, de los que sí pueden hacerlo, no eligen como destino otro país de la región, sino que prefieren Estados Unidos y Europa Occidental (UNESCO-IESALC, 2019).

Se podría pensar que la movilidad va a pasar a un segundo plano debido a la pandemia mundial y a las restricciones de viaje derivadas de la misma. Efectivamente, es más que probable que el próximo curso no vaya a haber estudiantes trasladándose a universidades de otros países. Una de las instituciones que atraen más alumnos de todo el mundo, la Universidad de Cambridge, ha anunciado que el próximo curso seguirá impartiendo en línea ${ }^{4}$. Del mismo modo, existe una importante controversia acerca de si las universidades de Estados Unidos, otro de los destinos favoritos de los estudiantes internacionales, no deberían renunciar a matricularlos el próximo curso, pues los mismos quedarían en una difícil situación si durante el otoño o invierno se produjera una segunda ola de contagios del COVID-19 (Mason, 2020).

No obstante, el hecho de que todas las instituciones de educación superior estén pasando su docencia a la modalidad a distancia, ofrece una oportunidad sin precedentes para la movilidad virtual. Si antes del COVID-19 el limitado número de programas impartidos a distancia en la región era un problema, ahora las posibilidades se han multiplicado. Asumiendo que, en el mejor de los casos, la docencia del próximo curso va a ser híbrida, ¿por qué no cursar todas o algunas de las asignaturas a distancia en una universidad de otro país? Es evidente que el alumno se va a ver privado de uno de los aspectos más importantes de la movilidad, que es la experiencia de vivir en otro país. Pero, sí que se beneficiará de algunas de las ventajas antes apuntadas, como son: la vivencia de otros estilos de aprendizaje; la mayor variedad de posibilidades de formación; $y$, una formación más personalizada según los intereses del alumno. Todo ello a un bajo coste, de manera segura y pudiendo compatibilizarlo con otras obligaciones (Reyes, 2012).

La movilidad, ya sea presencial o virtual, exige de estrechas relaciones de colaboración entre las universidades involucradas en la misma, que deben suscribir acuerdos interuniversitarios que garanticen el reconocimiento de los estudios cursados en la universidad de acogida. Es evidente que si en la región hay menos intercambios que en otras partes del mundo, es porque alumnos e instituciones se encuentran con serias dificultades a la hora de tramitar y concretar tales acuerdos. Como se ha apuntado antes, fueron los acuerdos adoptados en el marco del EEES los que dotaron a las universidades europeas de un esquema de trabajo y unas normas compartidas. Gracias a ese marco común, en cada curso académico se tramitan en torno a 340.000 movilidades de estudiantes entre más de 4.00o instituciones de educación superior de los 47 países pertenecientes al EEES.

En 1998 Francia, Alemania, Italia y Reino Unido firmaron la «Declaración de la Sorbona» con el propósito de crear un espacio compartido de Educación Superior. Un año más tarde fueron 29 países europeos los que suscribieron la «Declaración de Bolonia» que recogía, entre otros objetivos, el establecimiento de un sistema común de créditos para fomentar la comparabilidad de los estudios y promover la movilidad de estudiantes y titulados (los European Credit Transfer System o ECTS); y la adopción de un sistema fácilmente legible y comparable de titulaciones mediante el Suplemento Europeo al Título. Más adelante, en el Comunicado de Praga de 2001, se introducen otros elementos interesantes como es el desarrollo de sistemas de garantía de la calidad y de mecanismos de certificación y de acreditación, para incrementar el atractivo del EEES (MECD, 2003).

$4 \quad$ La universidad hizo pública su decisión el día 21 de mayo de 2020 a través de una comunicación de su Vicerrector, https://www.cam.ac.uk/coronavirus/news/update-from-the-vice-chancellor-18. 
Como se ha apuntado anteriormente, este proceso de Bolonia nace ligado a la necesidad de hacer efectiva una de las cuatro libertades del Mercado Común Europeo, la libertad de establecimiento. Pero, pronto trasciende este objetivo y se suman a la iniciativa países que no participan del mismo ni del proceso de integración europeo, hasta el punto, que el EEES está actualmente compuesto por 47 países europeos y la Unión Europea por 27. Se trata, además, de un proceso voluntario basado en una serie de declaraciones políticas suscritas por los ministros de educación de los países participantes, que no son jurídicamente vinculantes a pesar de que adaptarse al EEES requiere cambios legislativos.

La pertenencia a un espacio común de características similares es una decisión y un compromiso político que, como se ha apuntado, exige de reformas normativas, las cuales se justifican por los beneficios que se derivan de tener un sistema universitario que dialogue mejor y sea compatible con los de su entorno. Como se acaba de ver, el éxito del EEES no reside en el proyecto de integración europeo, al que muy pronto trascendió, sino en el valor añadido que tal iniciativa tiene por sí misma. Así lo reconocía el Ministerio de Educación de España, en un documento que sirvió como marco conceptual para impulsar las reformas que permitieron la integración del sistema universitario español en el EEES, y en el que se indicaba que los cambios que se iban a llevar a cabo eran, en realidad, «eficaces instrumentos orientados al logro de una mejora de la calidad y de una plena adecuación de estas enseñanzas» a las exigencias de una sociedad del conocimiento (MECD, 2003).

La matrícula universitaria en la región ha crecido muy notablemente en los últimos años, y la mayoría de los países se encuentran en la fase de masificación o universalización de esta etapa educativa. El gran reto para los responsables gubernamentales es ahora la calidad de la educación universitaria, porque de ello depende en gran medida que sus países sean capaces de superar la trampa de la productividad identificada por CEPAL. Por otra parte, es precisamente en esta línea hacia donde apuntan las tendencias internacionales, pues las universidades no solo compiten por estudiantes o por presupuesto, sino también por la calidad (Musselin, 2018). Lo que explica el éxito de los sellos internacionales de calidad, así como otras herramientas de medición más o menos precisas para la misma, incluyendo los siempre controvertidos rankings internacionales.

El otro factor apuntado por el Ministerio español, la adecuación de la oferta formativa a las demandas de una economía y sociedad del conocimiento, en el año 2020 es mucho más acuciante. Desde hace unos años se suceden los estudios e informes que advierten de los efectos que la revolución digital va a tener en el mercado de trabajo, y es muy posible que la pandemia haya acelerado el proceso. De hecho, la propia educación universitaria se está viendo transformada profundamente como consecuencia de la misma, al verse obligada a hacer un uso más intensivo de las nuevas tecnologías para seguir impartiendo clases e, incluso, investigando.

En los próximos años todos los países iberoamericanos van a tener que adaptar su marco normativo a la nueva realidad universitaria que se está fraguando en este tiempo de confinamiento y distanciamiento social. Por lo que es una excelente oportunidad para acordar una serie de medidas comunes que giren en torno al estudiante, que es el beneficiario último de todo proceso de convergencia de estas características, en la medida en que esto se traduce en más oportunidades para él, tal y como se ha visto en el caso del EEES. 


\section{3. ¿Qué está haciendo la OEI para construir un espacio iberoamericano de educación superior?}

Los apartados anteriores de este artículo han servido para explicar, en primer lugar, porqué la educación superior y la ciencia se han convertido en áreas estratégicas de una organización de cooperación como la OEI, con más de 70 años de experiencia sobre el terreno; y, en segundo lugar, para exponer los fundamentos de uno de los aspectos claves de su programa de educación superior, que es la creación de un espacio compartido de educación superior y ciencia. En el presente apartado vamos a presentar los elementos concretos de la arquitectura de dicho espacio en los que se está trabajando, y las iniciativas promovidas en cada uno de ellos. Es evidente que esta agenda de trabajo se ha visto afectada por la declaración de la pandemia global pero, como se ha argumentado, el objetivo sigue aún más vigente que antes, por lo que se está haciendo un esfuerzo por adaptar esa agenda a las nuevas circunstancias, para seguir avanzando en la misma.

\subsection{Transparencia y publicidad: refuerzo de los sistemas estadísticos}

La primera dificultad que nos encontramos a la hora de definir nuestro programa para 2019 y 2020 es que no contamos con suficiente información y datos sobre nuestros sistemas universitarios y científicos desde una perspectiva comparada, para saber exactamente el grado de convergencia que existe entre los mismos.

En la región iberoamericana existen dos redes de producción de datos estadísticos relacionados con el ámbito de este programa, la Red IndicES ${ }^{5}$ de producción de datos de educación superior y la red RICYT 6 de producción de datos de ciencia y tecnología, coordinadas por el OCTS-OEI. En concreto:

- IndicES: es la Red Iberoamericana de Indicadores de Educación Superior, iniciativa de cooperación regional para la producción de información estadística que involucra a los productores de información sobre educación superior en los países iberoamericanos, personal académico, expertos de organismos de cooperación regional y usuarios finales. En 2010 se realizó la primera reunión en Montevideo. El objetivo de la Red IndicES es la construcción de estadísticas comparativas de educación superior, consolidando un sistema regional de indicadores que releve y publique información estadística comparable en un marco de cooperación internacional, complementando los esfuerzos ya existentes. Se aspira a mejorar así las estadísticas de manera sostenida a lo largo del tiempo, reflejando las particularidades de la educación superior en Iberoamérica.

- RICYT: Es la Red Iberoamericana de Indicadores de Ciencia y Tecnología de la que participan las unidades responsables de la producción estadística de los ministerios de ciencia iberoamericanos. Este año cumple sus 25 años de existencia. Tiene el objetivo de promover el desarrollo y el uso de instrumentos para la medición y el análisis de la ciencia y la tecnología en Iberoamérica, en un marco de cooperación internacional, con el propósito de profundizar en su conocimiento y su utilización como instrumento político para la toma de decisiones.

$5 \quad$ http://www.redindices.org/

6 http://www.ricyt.org/ 
La RICYT, a lo largo de sus 25 años de trabajo, ha acompañado el desarrollo de las oficinas estadísticas de ciencia y tecnología en muchos países. Actualmente, releva un total de 137 indicadores. Gran parte de esas series están disponibles para la mayor parte de los países. En los últimos años se han incorporado indicadores de género, de servicios científicos y tecnológicos, entre otros. Por su parte, la Red INDICES, en su primer relevamiento recolectó 120 indicadores con una tasa de respuesta promedio del $40 \%$. Tres años después, recoge 127 series estadísticas con una tasa de respuesta del $45 \%$.

Estas redes aportan una información muy relevante para el fortalecimiento de las políticas públicas de educación superior y ciencia en Iberoamérica. Sin embargo, los propios países participantes de estas redes han mostrado la necesidad de mejorar su capacidad técnica para evaluar sus políticas públicas y recoger datos estadísticos de un mayor número de indicadores y de mejor calidad. Concretamente, aún persiste una fuerte heterogeneidad de capacidades en las oficinas estadísticas, que afectan a la calidad y comparabilidad de los datos.

En el seno de la Red IndicES se han creado una serie de grupos de trabajo con el objetivo de desarrollar nuevos indicadores o mejorar las definiciones de los que ya tiene la Red en áreas estratégicas, como son las de internacionalización y diversidad. El grupo de internacionalización está trabajando con algunos países iberoamericanos para el desarrollo de nuevos indicadores, para ofrecer más luz sobre cómo se están realizando las movilidades en la región y entre qué países, en el marco de programas específicos de movilidad con el fin de verificar el interés real por promover la internacionalización más allá de la de ciclo completo. También se está llevando a cabo una colaboración con la principal asociación de universidades privadas de la región, para poder incorporar la información procedente de este tipo de instituciones, cuya matrícula ha crecido de modo muy relevante en los últimos años.

Además, se ha creado un grupo específico para detectar qué países tienen más dificultades a la hora de recoger y/o analizar datos. Ésta es, sin duda, una cuestión clave para poder mejorar la información estadística disponible, y por ese motivo desde la OEI se está avanzando en un proyecto estratégico, con un componente consagrado al refuerzo de las capacidades estadísticas de estos países y de ambas redes en su conjunto.

Los indicadores son un valioso instrumento de evaluación de políticas públicas, tal y como se ha señalado, pero en el ámbito universitario también las instituciones de educación superior y sus programas formativos son sometidos a rigurosos procesos de evaluación de la calidad. De modo, que este primer punto del programa de la OEI enlaza directamente con otra de las áreas claves necesarias para construir ese espacio compartido al que antes se ha hecho referencia, que es el de la calidad.

\subsection{Hacia un modelo armonizado de garantía de la calidad}

En el apartado segundo se ha explicado que una iniciativa tan ambiciosa como el EEES nació al albur del Mercado Común, que se apoya en la libre circulación de bienes, personas, capitales y servicios. Para lograrlo fue necesario remover las fronteras, pero también otros obstáculos no físicos. En especial, normativas que en la práctica impedían que un producto francés pudiera ser vendido en Alemania. Éste fue el supuesto que dio pie a la célebre sentencia Cassis de Dijon del Tribunal Europeo de Justicia, en la que se consagró el principio de mutuo reconocimiento: cualquier producto fabricado y comercializado en un Estado miembro conforme a las normas de ese Estado, debe ser admitido en cualquier otro país europeo. En definitiva, este reconocimiento descansa en la confianza mutua, en 
la convicción de que todos ellos aplican normas similares a la hora de producir y comercializar sus productos y que estos son, por tanto, de la misma calidad.

Las evaluaciones externas a las que se someten los programas e instituciones universitarias de la región son los llamados a generar esa confianza mutua entre nuestros sistemas, indispensable para lograr que haya más intercambios y más cooperación entre las universidades iberoamericanas. De acuerdo a lo expuesto, cuanto más similares sean los procedimientos de evaluación y los estándares que estas agencias utilicen, habrá más probabilidades de incrementar esa confianza. Por este motivo, la OEI reconoce en los organismos de acreditación de la calidad iberoamericanos unos actores fundamentales del proyecto en educación superior.

Un ejemplo de ese trabajo es el Informe de Aseguramiento de la Calidad de la Educación Superior en Iberoamérica que la OEI, a través del Observatorio, está realizando con la Comisión Nacional de Evaluación y Acreditación Universitaria (CONEAU). En este informe se reúnen la descripción y comparación de cada sistema de aseguramiento de la calidad de la educación superior a partir de la información recabada disponible, así como de entrevistas a referentes clave de los países para conocer sus actuales modelos de evaluación de la calidad.

La OEI aspira a la aproximación de esos modelos de evaluación, y que los mismos se desarrollen de acuerdo a unos procedimientos y estándares comunes. Para ello, y teniendo en cuenta que los sistemas de aseguramiento externo de la calidad de la región en términos generales cuentan ya con un notable grado de madurez, la OEI ha comenzado por trabajar en ámbitos específicos que requieren sus propios criterios de calidad.

Es el caso, por ejemplo, de las enseñanzas artísticas, cuyas propias características exigen de un modelo y de unos indicadores de evaluación distintos. Por ejemplo, son disciplinas que necesitan de unas instalaciones especiales para ser impartidas (escenarios, salas de ensayo, talleres de pintura o escultura, etc.), y también de un perfil de profesorado particular, con experiencia académica y docente pero que, a su vez, sean creadores. Todo ello se plasmó en la Declaración por una evaluación y acreditación de la calidad específica de la Educación Superior en Artes en Iberoamérica, elaborada y suscrita por la OEI y las Universidades de Arte en Guayaquil el 31 de octubre de 2019, documento de cierre del I Encuentro Iberoamericano para la evaluación de la calidad en la Educación Superior en Artes.

El día en que la Organización Mundial de la Salud declaraba la pandemia mundial por el Covid-19, la OEI clausuraba el Seminario Iberoamericano de Calidad en la Educación a Distancia, organizado junto al Consejo de Aseguramiento de la Calidad de la Educación Superior de Ecuador (CACES) y la Universidad Técnica Particular de Loja (UTPL). En este evento se dieron cita instituciones de educación superior (IES) con amplia experiencia en esta modalidad de impartición, que compartieron sus mejores prácticas con el resto de universidades participantes, y agencias de calidad de distintos países de la región. Todas ellas están actualmente adaptando sus modelos y criterios de evaluación a este tipo de enseñanza, cuya matrícula no deja de crecer. En concreto, ha experimentado un incremento del 73 \% desde 2010, mientras que la presencial lo hizo un $27 \%$. En 2010, casi dos millones y medio de los 21 millones de estudiantes universitarios de primer título en Iberoamérica estudiaban a distancia, representando un 11,7 \% del total. En 2017 esta modalidad de enseñanza representó un 15,3 \% del total y abarcó a 4 millones trescientos mil alumnos (OCTS-OEI 2019b). Por ello en esta situación internacional de emergencia sanitaria en la que las instituciones de educación superior están adaptando a marchas 
forzadas su formación a modalidades virtuales o a distancia, cobra especial relevancia la importancia de asegurar la calidad de dichas metodologías.

Para la preparación de este Seminario, la OEI constituyó un Grupo de Trabajo con diversas agencias de calidad iberoamericanas con el objetivo de poner en común y comparar los indicadores que están utilizando para evaluar las titulaciones a distancia. Con ocasión del Seminario, este Grupo de Trabajo se reunió para acordar una serie de definiciones y estándares comunes, que se recogen en la Guía Iberoamericana para la Evaluación Externa de la Calidad de la Educación a Distancia, recientemente publicada (OEI, 2019).

Es la primera vez que la región cuenta con un documento de estas características, que ofrece a los organismos garantes de la calidad y a las propias universidades una serie de indicadores claros para medir la calidad de las enseñanzas impartidas en modalidad a distancia o virtual. Si bien esta Guía de la OEI entiende que la concreción de cada uno de esos indicadores corresponde a las agencias nacionales, que son las que mejor conocen sus respectivos sistemas universitarios. A modo de ejemplo, la Guía Iberoamericana señala qué aspectos tener en cuenta a la hora de determinar la ratio profesor/alumno para que todas las agencias puedan establecer la misma conforme a unos mismos criterios, pero es cada agencia la que decide cuántos alumnos debe haber por profesor.

En conclusión, esta Guía no pretende sustituir a las desarrolladas por cada agencia, sino ofrecer orientaciones comunes a todas ellas que faciliten el reconocimiento mutuo de las evaluaciones que realicen conforme a esos criterios compartidos. En este caso concreto el ejemplo europeo ha estado más que presente y, de hecho, dos de las personas participantes en este Grupo de Trabajo a su vez formaron parte del Grupo de Trabajo de la European Association for Quality Assurance in Higher Education (ENQA) que elaboró una guía similar para evaluar la educación virtual en el EEES.

\subsection{Movilidad y reconocimiento de estudios}

Como ya se ha comentado anteriormente, la baja movilidad académica de una región capaz de entenderse en dos lenguas en un contexto en el que no cesa de aumentar el número de estudiantes y profesores que realizan intercambios con universidades de otros países solo se explica debido a la existencia de serios obstáculos. Entre los mismos, cabe destacar especialmente las dificultades a las que se enfrentan los estudiantes para que sus universidades reconozcan los periodos de estudios realizados en otro país. Desde la OEI, donde se tiene una amplia experiencia en programas de movilidad, se apuesta decididamente por el desarrollo de una métrica común para facilitar ese indispensable reconocimiento de estudios.

El desarrollo de una métrica iberoamericana debe partir de una premisa, y es que tiene que ser totalmente compatible con los ECTS de España y Portugal. De este modo, en los últimos años en la región se han desarrollado diversos modelos de créditos académicos bajo denominaciones distintas. Son un importante referente a tener en cuenta para elaborar a partir de los mismos una propuesta común, pero en la definición exacta de esa propuesta hay que tomar en consideración diversos aspectos.

En primer lugar, en algunos países se miden las actividades académicas, pero no el trabajo autónomo del alumno que está en la base del ECTS, puesto que todo el proceso de Bolonia sitúa al estudiante en el centro del mismo. En aras a esa compatibilidad antes mencionada, resulta indispensable que el modelo de métrica común iberoamericana llegue a un acuerdo acerca de cómo definir y medir la dedicación del alumno a la titulación fuera del horario de clases y prácticas. En segundo lugar, se aprecia también la escasa 
implantación de muchos de esos modelos, lo cual advierte de las dificultades que las universidades pueden estar encontrando a la hora de adaptar su ordenación académica a los mismos. Por otra parte, también se debe reconocer que la internacionalización no es una apuesta estratégica de todas las universidades iberoamericanas. Es más, algunas de ellas ni siquiera cuentan con residencias o alojamientos para estudiantes, pues han sido creadas para atender la demanda de educación universitaria de su entorno más próximo. En dicho caso, su interés por adoptar un sistema de métrica común es muy escaso, porque no aspiran a captar alumnado foráneo. Aunque la movilidad virtual -antes mencionada- puede hacerles cambiar de opinión y abrirles nuevas oportunidades. En la OEI se espera que así sea, porque ya se han señalado las múltiples ventajas que se derivan de la internacionalización de los estudiantes, incluso de la llamada internacionalización en casa, esto es, de la convivencia en el mismo campus con alumnos de otros países, incluso aunque esta se produzca en el campus virtual. Es una manera muy efectiva de disfrutar de todo lo que implica formar parte de la comunidad iberoamericana.

Por ello, la OEI lanzó recientemente una convocatoria de prácticas de calidad de internacionalización, para que las universidades presenten sus mejores experiencias y programas en este ámbito, incluyendo internacionalización virtual. El propósito es animar a todas las universidades a que adopten programas de movilidad para sus estudiantes y profesores, especialmente ahora que los mismos se pueden adaptar a todo tipo de perfiles y posibilidades de financiación. Esta iniciativa es compartida con el Instituto Internacional para la Educación Superior en América Latina y el Caribe (UNESCO-IESALC), la Universidad Autónoma de Bucaramanga en Colombia (UNAB), la Universidad de Alcalá en España (UAH), la Universidad Nacional de Córdoba (UNC) como anfitriona del Centro Regional para la Educación Superior en América Latina y el Caribe (CRES) y la Asociación Colombiana de Universidades (ASCUN), constituyendo un Comité de Selección con expertos que se encargarán de elegir las mejores prácticas de internacionalización de las universidades iberoamericanas.

Esas buenas prácticas que deberían abordar todos los aspectos relacionados con la internacionalización de una IES:

1. Políticas y planes institucionales de internacionalización. Se considerarán aquellas prácticas asociadas o derivadas de la publicación y/o aprobación por parte de la institución de políticas y/o planes institucionales de internacionalización con impacto en el conjunto de la institución, alguno de sus centros o en su comunidad académica.

2. Movilidad entrante y saliente (de estudiantes /profesores/personal de administración). El compromiso de la institución, sus distintos centros y su comunidad académica con la movilidad de estudiantes, personal docente e investigador y personal de administración podrá ser reflejado a través de muy diversas prácticas. Desde planes propios de financiación de movilidad internacional, existencia de convenios bilaterales con reconocimiento académico que permitan la movilidad académica, cifras de movilidad entrante y saliente diversificada (no focalizada a un único destino/ país/socio), programas de profesores e investigadores internacionales visitantes, programas de becas y servicios de orientación y apoyo a la movilidad, entre otros.

Se incluye una mención especial para aquellos programas de movilidad diseñados y/o adaptados para personas con discapacidad. 
3. Internacionalización del currículo - Interculturalidad, programas compartidos. Acciones que fomenten la internacionalización del currículo mediante el diseño de currículos formativos que incorporen competencias internacionales e interculturales en las metodologías docentes y en los contenidos; la Internacionalización en casa (Internationalization at Home), la implementación de titulaciones conjuntas (Joint Degrees) con otras universidades iberoamericanas, el impulso de intercambios virtuales, la docencia en otras lenguas, entre otras. Como consecuencia de la pandemia, en la convocatoria se ha hecho especial énfasis en la búsqueda de ejemplos de movilidad virtual.

4. Proyectos de investigación e innovación colaborativa. Podrán incluirse prácticas de innovación e investigación participativa y multidisciplinar de carácter internacional; prácticas que pongan en valor la transferencia de conocimiento y de resultados de investigación y la transformación social en el contexto iberoamericano; prácticas que dinamicen la investigación interactiva entre universidad-empresasociedad civil y administración/gobierno en Iberoamérica; prácticas que promuevan el trabajo en equipo, el intercambio y los proyectos de grupos d investigación de distintos países, entre otros.

5. Programas e iniciativas internacionales de extensión y programas de cooperación para el desarrollo. Se podrán incluir en esta sección aquellos proyectos de cooperación Sur-Sur dedicados al ámbito de la educación (ODS 4) y al desarrollo sostenible en alianza con otras universidades y/o instituciones (ODS 17). También se podrán incluir prácticas de calidad relacionadas con la extensión universitaria de dimensión internacional: actuaciones formativas y culturales de extensión que posean carácter internacional y donde se promueva la proyección de la universidad a la sociedad y su desarrollo profesional. Se valorarán prácticas dedicadas a la formación continua y a la formación a lo largo de la vida (life long learning) con dimensión internacional.

Esas mejores prácticas se recogerán en un Manual Iberoamericano de Buenas Prácticas de Internacionalziación (MIBPI) y se presentarán en un seminario, que se va a celebrar en noviembre en Bogotá en formato virtual, para poder cumplir con las exigencias sanitarias derivadas de la pandemia de COVID-19.

Este seminario servirá también de espacio de encuentro y reflexión sobre ese modelo iberoamericano de métrica común, tan necesario para la región, que servirá para propiciar una mayor movilidad de estudiantes, pero también para contribuir a agilizar el reconocimiento de títulos de los egresados universitarios.

\section{Universidad Iberoamérica 2030}

La puesta en marcha del proyecto de educación superior de la OEI, de acuerdo a lo previsto en su Programa-presupuesto, se acompañó de un proceso de diagnóstico del sistema universitario iberoamericano (OEI, 2018). Ello ha permitido ajustar y adaptar las acciones antes señaladas a las verdaderas necesidades de la educación superior en la región, ha abierto un proceso de diálogo con los principales actores (universidades, estudiantes, agencias de calidad, etc.) y ha servido de base para diseñar la estrategia Universidad Iberoamérica 2030, fundamento del próximo programa-presupuesto. 
El hecho de que la declaración de la pandemia coincidiera con la celebración del seminario de la OEI sobre calidad en la educación a distancia, es la prueba más evidente de que este diagnóstico ha sido de lo más acertado, y que realmente estamos en disposición de ser útiles ante el nuevo escenario que se va a abrir.

Iberoamérica ya ha cruzado la barrera de los 30 millones de estudiantes universitarios. Según datos del Informe Diagnóstico de la Educación Superior en Iberoamérica (Sainz y Barberá, 2019), esta expansión de la matrícula se explica porque cada vez hay más estudiantes universitarios de primera generación, esto es, jóvenes que proceden de entornos desfavorecidos que son los primeros de su familia que acceden a la universidad. Por otra parte, a esos futuros egresados universitarios les espera un mercado de trabajo en profunda transformación, que no solo demanda trabajadores más cualificados sino, además, con sólidas competencias tecnológicas y digitales; capacidad de innovar y emprender; y, de adquirir y actualizar constantemente su conocimiento.

En este Informe se pone también de manifiesto, que en América Latina y el Caribe el porcentaje de asistencia a la educación superior aumenta según se incrementa el nivel de riqueza. Sin embargo, la región se encuentra en porcentajes alentadores en los niveles bajos de riqueza, aunque alejados todavía de la relevancia que estos porcentajes adquieren en otras regiones como Europa o América del Norte. Tal y como se señala en el informe (Sainz y Barberá, 2019):

Visto por niveles de riqueza, solo el $6 \%$ de los más pobres asiste a educación superior, este porcentaje asciende al $11 \%$ para los pobres y al $16 \%$ para la clase media. Los ricos por su parte logran que el 28 \% de sus jóvenes se encuentren dentro de la educación superior y los más ricos ya se sitúan en el 50 \%. La media estaría en el $21 \%$ (p. 35).

Asimismo, el número de universidades también ha crecido en estos años para dar respuesta a este crecimiento de la demanda, y actualmente en la región hay 3.724 universidades. Como se ha apuntado anteriormente, el gran desafío de nuestros sistemas universitarios ahora es garantizar la calidad de la formación que se imparte en todas esas IES. El profesorado es siempre uno de los factores más importantes en cualquier evaluación de calidad, porque de ellos depende en gran medida que los alumnos adquieran las competencias y resultados académicos esperados. A este respecto, cabe señalar que el porcentaje de profesores con doctorado en las universidades de América Latina está por debajo del $12 \%$, salvo en Brasil.

Por este motivo, dentro de la estrategia de la OEI Universidad Iberoamérica 2030, el programa Paulo Freire Plus ( $\mathrm{PF}+$ ), acción de movilidad académica vinculada a la investigación, ocupa un puesto destacado. El objetivo de esta iniciativa es fomentar la vocación científica, y también apoyar el trabajo de los grupos iberoamericanos de investigación, que a menudo carecen de los recursos que su trabajo necesita y merece.

El programa $\mathrm{PF}$ + financia becas de doctorado a graduados y profesores universitarios que quieran cursar sus estudios doctorales en una universidad de la región distinta de aquella en la que han realizado sus estudios de grado y master, o en la que desarrollan actualmente su labor docente. De este modo, el PF+ contempla dos modalidades: investigador junior y senior.

La modalidad junior implica realizar todo el doctorado en una universidad de otro país de la región, lo que contribuye a la creación de vínculos sólidos entre universidades (les permite compartir proyectos de investigación, publicaciones, etc.) y la creación de redes temáticas entre universidades. La investigación es una actividad muy intensiva en recursos, por lo que la creación de dichas redes contribuirá a que la investigación 
conjunta que se realice en el seno de las mismas, tenga masa crítica suficiente como para tener impacto regional y/o global. El alumno tiene un plazo de tres años para completar su doctorado en esta modalidad.

La modalidad senior permite al profesor universitario compaginar su formación doctoral con su carrera docente y su vida personal. De este modo, el doctorando tan solo tendría que estar en la universidad de destino en la que cursa el doctorado el primer año de sus estudios (tiempo imprescindible para integrarse en el equipo de investigación, definir su tema de investigación y comenzar esta). Los otros tres años que tiene para completar el doctorado estaría trabajando en su tesis en su universidad de origen, compatibilizando la misma con su trabajo docente (de ahí que se le conceda un año más que en la modalidad junior para terminar la tesis).

En el año 2019 se ha lanzado con éxito un programa piloto financiado por la Junta de Andalucía, el programa presidencial Becas Honduras 2020 y la OEI. El proceso de selección y adjudicación se ha realizado de manera competitiva y de acuerdo a los méritos de los candidatos. En él se han recibido 300 solicitudes para 9 becas, lo que demuestra el enorme interés y expectativas que ha suscitado el programa. Colombia ha sido el país de origen de la mayoría de los candidatos, y España el destino más elegido entre los mismos. Las áreas de conocimiento en las que se han concentrado gran parte de las postulaciones han sido Ciencias Jurídicas y Educación, mientras que las Ingenierías apenas recibieron un 1 \%.

Otro de los objetivos que aborda esta estrategia, además de la escasa movilidad y la necesidad de robustecer los sistemas internos y externos de garantía de la calidad ya comentados anteriormente, es la de potenciar la misión investigadora y de vinculación de la universidad con la sociedad. El $57 \%$ de los investigadores iberoamericanos realizan sus actividades en el ámbito universitario (RICYT, 2019). Si se tiene en cuenta que menos del $12 \%$ de los profesores universitarios de la región son doctores, se puede afirmar que, a día de hoy, Iberoamérica no tiene capacidad investigadora suficiente para generar prosperidad y crear dinámicas de desarrollo y crecimiento alineadas con las exigencias de una economía global y del conocimiento. Después del pasado mes de marzo sabemos, además, que esta ausencia de músculo investigador representa también un riesgo para la salud y el bienestar de nuestras sociedades.

Además, en el Informe sobre los Investigadores Universitarios y su Vínculo con el Entorno en América Latina (OCTS-OEI, 2019c), se pone de manifiesto la fragilidad de los vínculos entre las instituciones académicas y su entorno. Dos terceras partes del personal investigador encuestado desarrolló alguna actividad de vinculación, formal o informal, durante el año 2018, pero tan solo dedicaron el $12 \%$ de su tiempo a actividades relacionadas con el entorno y, de ese ya de por sí bajo porcentaje, dedicaron un $6 \%$ a divulgación científica y otro $6 \%$ a actividades de vinculación con la sociedad propiamente dichas.

En consecuencia, también se hace imprescindible potenciar las actuaciones de vinculación de las universidades con el entorno productivo y social, impulsando el desarrollo de herramientas metodológicas para la gestión y planificación de estas actividades en las universidades, así como permitir a las administraciones públicas diseñar e implementar políticas que brinden a la sociedad datos que la aproximen a la universidad como prestadora de servicios. Esto equivale también a coproducir conocimiento, transmitirlo e incorporarlo en la sociedad, es decir, a estimular la transferencia del conocimiento entre universidad y entorno, de modo que la investigación desarrollada en el seno de las universidades tenga un provecho económico y social y contribuya a fomentar y potenciar el bienestar de la sociedad. 
Esta noción amplia que defiende la OEI del concepto de transferencia del conocimiento, entendiendo que el mismo puede tener un impacto social positivo además o aparte del económico, explica esta apuesta porque los Objetivos de Desarrollo Sostenible (ODS) de la Agenda 2030 sean el marco de referencia fundamental de la investigación iberoamericana. En nuestra región se concentran muchos de los desafíos a los que pretende dar respuesta la Agenda 2030. Razón por la cual tiene especial sentido que el desarrollo de nuestra capacidad investigadora en las distintas disciplinas se oriente a temáticas relacionadas con los ODS.

Los ODS adquieren aún más significado si cabe en situaciones de emergencia como la actual, como marco de actuación para abordar las secuelas que perdurarán en el futuro tras el COVID-19; o en posibles nuevas emergencias de cara a transformar los sistemas productivos y sociales iberoamericanos para que estos resulten más justos, equitativos, sostenibles y resilientes.

Por todo ello, la OEI entiende que estos esfuerzos por crear en Iberoamérica ese espacio compartido de educación superior e investigación deben alinearse con la Agenda 2030 y sus ODS, los cuales nos indican incluso la mejor manera de llevarlos a cabo. Desde esta organización se aboga muy activamente por las alianzas estratégicas del ODS 17 , pero estas van a resultar aun más imprescindibles en el panorama económico y social tan complicado que está dibujando la pandemia.

La retracción económica del último lustro ya suponía un riesgo para los sistemas científicos y tecnológicos iberoamericanos, por el desequilibrio cada vez más acentuado entre un número creciente de investigadores y la escasez cada vez mayor de recursos. El gasto en $\mathrm{I}+\mathrm{D}$ por investigador en Iberoamérica es, en Paridad de Poder de Compra (PPC), de 182.720\$. Una cifra sensiblemente inferior a la media mundial (242.258\$) y que supone tan solo un 60 \% de la cifra de inversión en I+D por investigador de Europa y Norteamérica hace en I+D (295.866\$) (OCTS-OEI, 2020).

Las previsiones económicas para el presente año han empeorado de manera extraordinaria como consecuencia de la pandemia mundial de COVID-19, señalando que los países de la región están entrando en una grave recesión económica. CEPAL prevé una caída del 5,3\% del PIB en América Latina y, sobre todo, un gran incremento de la pobreza. Ante tal escenario, resulta indispensable adoptar medidas que eviten un retroceso o la destrucción de gran parte de las capacidades científicas que se han ido construyendo en América Latina en las últimas décadas. Para evitar que así sea, será necesario la concertación y el compromiso de muchos: gobierno y administración; universidades y su personal investigador; empresas y sociedad civil.

En resumen, resulta imprescindible trabajar para promover en nuestra región una educación superior e investigación de calidad, capaces de alcanzar una dimensión regional al servicio de la construcción de una ciudadanía con sentido crítico, que tome decisiones sobre la base de evidencias, que permitan transformar los sistemas para que estos sean más prósperos, más justos y más sostenibles. A todo ello aspira la estrategia Universidad Iberoamérica 2030 de la OEI, un ambicioso plan de trabajo para la próxima década que va a comenzar su marcha en un bienio que promete ser extremadamente difícil. Los 70 años de la OEI han sido testigos de otras épocas igualmente complicadas. La lección aprendida de las mismas es que tendremos que redoblar nuestras fuerzas para poder avanzar lo máximo posible en un proyecto que es en sí mismo una potente vacuna contra emergencias como la que padecemos y sus temidas consecuencias. 


\section{Referencias}

CEPAL (2020). El desafío social en tiempos del COVID-19, Informe especial Covid -19. Recuperado dhttps://repositorio.cepal.org/bitstream/handle/11362/45527/ S2000325_es.pdf?sequence=5\&isAllowed $=y$

Comisión Europea (2019). Erasmus + Higher Education Impact Study, DirectorateGeneral for Education, Youth, Sport and Culture.

Mason, T. (2020). Is it ethical to take in foreign students mid-crisis?, University World News. Recuperado de https://www.universityworldnews.com/post. php?story $=202005141501467$

MECD (2003), La integración del sistema universitario español en el Espacio Europeo de Enseñanza Superior. Documento-marco., Ministerio de Educación, Cultura y Deporte de España. Recuperado de http://www.eees.es/pdf/DocumentoMarco_10_Febrero.pdf

Musselin, C. (2018). New forms of competition in higher education. Socio-Economic Review, 16(3), 657-683.

OCDE et al. (2019). Perspectivas económicas de América Latina 2019: Desarrollo en transición, OECD Publishing, Paris, https://doi.org/10.1787/g2g9ffia-es

OEI (2018). Programa-presupesto 2019/20. La Antigua: OEI. Recuperado de https:// bit.ly/2MZkMyA

OEI (2019). Guía Iberoamericana para la Evaluación Externa de la Calidad de la Educación a Distancia. Recuperado de https://bit.ly/3huOGsw

OCTS-OEI (2019a)- Panorama de la Educación Superior en Iberoamérica, Papeles del Observatorio, 12 1-68.- Recuperado de https://observatoriocts.oei.org.ar/wpcontent/uploads/2019/11/Papeles-12-Web.pdf

OCTS-OEI (2019b). Se expande la educación superior a distancia en Iberoamérica , aunque todavía es incipiente, Informe de coyuntura $n^{\circ}$ 6. Recuperado de http:// octs-oei.org/coyuntura/coyunturao6.pdf

OCTS-OEI (2019c). Los investigadores universitarios y su vínculo con el entorno en América Latina, Papeles del Observatorio, 11, 1-34. Recuperado de https://bit. ly/2Bb6DMo

OCTS-OEI (2020). ¿Cuánto puede expandirse la cantidad de investigadores en Iberoamérica?, Informe de coyuntura, 7, 1-4. Recuperado de http://octs-oei.org/ coyuntura/coyunturao7.html

Reyes, S. (2012). Las experiencias educativas de movilidad virtual de los estudiantes de formación de postgrado: un estudio del caso UNAB, Seminario de Iniciación a la Investigación en Educación y TIC, Máster oficial en Educación y TIC 2012, de la Universitat Oberta de Catalunya (UOC). Recuperado de http://openaccess.uoc. edu/webapps/o2/bitstream/10609/36822/7/sreyesgTFMo612memoria.pdf 
RICYT (2019). Informe sobre el Estado de la Ciencia en Iberoamérica. Buenos Aires: OEI. Recuperado de http://www.ricyt.org/wp-content/uploads/2019/10/edlc2019.pdf

Sainz, J. y Barberá, R. (2019). Diagnóstico de la Educación Superior en Iberoamérica, Madrid: OEI. Recuperado de https://bit.ly/2Y3IEaV

UNESCO-IESALC (2019), La movilidad en la Educación Superior en América Latina y el Caribe: retos y oportunidades de un Convenio renovado para el reconocimiento de estudios, títulos y diplomas. Caracas: IESALC 